\title{
Public Health And Population Perspective Of COVID-19 As A Global Pandemic
}

\author{
Nazneen Akhter, M. Salim Uzzaman ${ }^{\mathrm{b}}$, Amr Ravine
}

\begin{abstract}
:
COVID-19 appeared as an infectious disease of global health emergency and highest public health concern of 21 st century for this world due to its high speed of spread across the globe. The disease started as one single case to a cluster of cases in Wuhan, China (Dec 2019) and within few months with its continuous upsurge of cases spreading globally which has created enormous threat and tension across the global Public health care field. Most significantly to share the fact that, this disease caused high level of Risk Group mortality, high morbidity, health care services burden, panic anxiety, mental trauma and tension, social and economic insecurity, which also collectively surfaced by diverse range of social reaction and political pressure across the world. Also with the appearance of new and unknown pathogenesis of the disease has created the most and significant attention and concern for the scientific community and political leaders as well. The disease also varies significantly with its pattern of virus, sign, symptoms, characteristics including its epidemiological and public health response (like prevention strategy, diagnoses, case management, treatment pattern) across the countries. However, this variance is comparatively less rather even more in commonality while it comes to basic public health prevention interventions like frequent hand washing, wearing face mask, maintaining social/physical distancing (2 - 6 meter in between), individual isolation and community quarantine for suspected exposure and lockdown in community areas where cases are identified. These are basically the most commonly practiced public health interventions to deal with this disease in majority of the epicenters across countries. Also with the progression of the disease with its diverse categorical appearance of sign symptoms which are uniquely portraying this disease is more of a kind of COVID-19 syndrome rather than as COVID-19. Moreover, various factors including socio economic status, health status, population dynamics, health system and infrastructure, health behavioral pattern, nutrition and food habit and also access to information and knowledge made this viral disease one of the historically counted on expensive disease of the modern world to fight for. Specially to mention here, the case fatality rate distinctively vary with the population dynamics and the health system infrastructure and ability factors which have already created clear evidence as explained in this paper how these factors are uniquely distinguished and different from country to country. Apart from the contextual differences, the various kind of ongoing preventive measures (like prevention, diagnosis and treatment) learnt every day from the emerging characteristics and pattern of this viral disease which are most commonly practiced across countries with some kind of variance. According to the Public health prevention practice and interventions, the total world is quite connected and learning from each other's public health experiences to fight for the virus. However, the whole world community are eagerly waiting to see the result and outcome of the ongoing therapeutics and vaccine trial initiated in several countries, the people of the world are quite optimistic and hopeful that, global scientific community will be able to invent some miraculous and magic solution in upcoming months which ultimately will free the world from this most terrifying COVID-19 pandemic of 21st century.
\end{abstract}

Key Words: COVID-19, Risk Group, Syndrome, Community Quarantine, Population dynamics, Social
distancing 
Coronavirus Disease 2019 (Covid-19), is one of a historical astound of an infectious disease pandemic outbreak which made a rapid spread in a month from its first infection (index case ) inextricably turned into Global Pandemic affecting peoples worldwide with serious health burden and economic blow. ${ }^{1,2,3,4}$ From an epidemic and social realization point of view it's evident that, over the years we have been investing more on human information technology but less on scientific preparation of any Global Human disease threat of international concern and this realization was echoed by leadership of diverse discipline over the past many years in their articles , paper sharing and hard talk. In this COVID pandemic reality , while every country is dealing with an epidemic puzzle, at the moment this dichotomy of investment realization become more pertinent issue to look back and find thoughtful way forward solution for this world where every country is willing to make sensible and synchronized allocation of science, technology, research and health science so that, this COVID-19 kind of reality never happen to be an repeat of experience unlike past several infectious disease of global pandemic nature.$^{2,3}$ In a sense the world was least prepared to deal with any type of infectious disease global pandemic even when already experienced several type of global upsurge of particular and distinctive nature of infectious disease blow to this world in a time scale of every century, that means its quite known facts for many social scientist and epidemiologist that pandemic happens after almost every '100-years'. ${ }^{2,5}$

If we look back to the global epidemic time series analogy, the global outbreak history of 'The Great bubonic Plague of Marseille", in France 1720, 'Cholera pandemics', and its fast speed spread in India to Southeast Asia, the Middle East, Europe, and Eastern Africa in 1820, and in nineteenth century epidemic stagger of 'Spanish flu' or 1918 flu pandemic (influenza caused by an H1N1 virus) killing more of a 50 million people worldwide in $1920 .^{2,5,6}$

Taking a look at the emerging history of this disease, in late December 2019, Chinese health commission realized that an outbreak of pneumonia associated with Coronavirus disease 2019 (Covid-19) has occurred in Wuhan, China, and rapidly spreading to other parts of China and the world and reported that to WHO.,4 To see more scientifically from the genome sequencing of this virus, its pattern, type and virulence according to the virology and epidemiological evidence and scientific purviews, till date seven types of Corona virus have infected humans of which four cause common cold but other three are responsible for epidemic (SARS-CoV, MERS-CoV \& presently SARS CoV-2). 1,5, 6,78 In January 2020, from the genome sequence done by Chinese scientific community shared that the new virus responsible for Covid-19, cause severe acute respiratory syndrome Coronavirus 2 ( $\beta$-SARS-CoV-2) . The virus has been mostly detected in Nasopharyngeal secretion, throat, lower respiratory tract, stool, gastrointestinal tract, saliva, and urine samples and even in semen ${ }^{6,7}$

a. Dr. Nazneen Akhter, Founder \& Managing Director, VAALO avant-garde Ltd. Assistant Professor, Adjunct, North South University, Dhaka, b. Dr. M. Salim Uzzaman, Principal Scientific Officer, IEDCR, Dhaka, c. Amr Ravine Post Graduate Fellow, University of Technology, Sydney. Australia. 
Most significantly to share the fact that, this disease created so high level of mortality in 'Risk Group', high morbidity causing health care services burden, Public Panic , mental trauma anxiety, social and economic insecurity which are being surfaced by diverse range of social reaction and political pressure across the world that this disease could create the most and highest attention of the current world scientific community response which evidenced by the fact that, there are over 6000 and more articles on Coivd-19 sharing updated information till date.

While making a scientific trail of the disease symptomatic appearance and physical dynamics , initially the diseases presentation was more of respiratory tract infection associated with fever, dry cough and respiratory distress, but now it's evident that it can cause cytokine storm, thrombo-embolic manifestation in the vessels, lung injury, kidney injury, Cardio-vascular failure and eventually multiorgan failure $^{.910}$ There are instances where, limited number of children presented with post infection multi-organ inflammatory syndrome including skin manifestation, e.g. 'Covid-toe ${ }^{9,10)}$. The medical and scientific stun of this disease dynamics is as such that newer information are added every day as with the progress of science. Till day many epidemiological evidence confirmed that Covid19 has the characteristic of human-to-human transmission, predominantly through respiratory droplets and contact, but we are yet to confirm the secondary host between human and bats.

Deeper look to the disease from the scientific evidences and population dynamics on its sign symptoms points of view the global information-data till date tells us that, there are asymptomatic cases, pre-symptomatic cases and symptomatic cases ${ }^{11 .}$ But most of the cases $(80 \%)$ present with mild symptoms, which don't need hospitalization or oxygen therapy and can be treated at home. Some people present with moderate symptoms (20\%) associated pneumonia, becomes seriously ill and develops difficulty breathing and require hospital care. Recently CDC added some more list of symptoms for the coronavirus: chills, muscle pain, headache, sore throat, repeated shaking with chills and a loss of taste or smell. Only 3-5\% of the Coronavirus infection cases develop serious illness and need "intensive Care (ICU)" support and necessary urgent Medical intervention at the hospital $^{9,10,11}$. The clear scientific knowledge around the facts yet to understand why the Covid-19 infection is deferent like this in different people? However, the inference drawn on our body immunity and response is quite clear till today based on a huge number of case management and scientific observation across the world, that this immunity has a major role to play beside the amount of infectious dose. Moreover, there is another distinctive observation made through which is 'Group risk' like Obesity, Hypertension, Cardiac Diseases, Diabetes, smoking habits etc. and old age above 65 years were also important risk factors influencing the severity of disease progression and mortality. ${ }^{12,13}$ However, it is by now become more clarified with the facts and evidences around the sign symptoms about the disease which have been observed and recorded so far and give us a clear hint on the nature of this disease and its symptomatic appearance which in most case collective like syndrome rather than few symptoms of respiratory tract infection. The disease at initial stance may appear with few symptoms but in most case make very rapid progression to multiple organ involvement, especially if remain undiagnosed or untreated, so "COVID-19 syndrome can be its another synonym appropriate to consider in future". 
While to see the geographical dynamics and to relate this disease science, Wuhan, China was the first epicenter of this pandemic before it stated spreading to other Asian countries, south Asia, middle east (Iran the epicenter), Europe (Italy the epicenter) and Spain (western Europe epicenter) and then to USA (NY the epicenter). ${ }^{1,3,4}$ But the spread, number of cases and case fatality rate (CFR) are different in different countries and region of the globe, which has created high level of concerns in the global communities, countries and scientific arena. ${ }^{4,11}$ Moreover, the case fatality rate (CFR) also widely varies across the countries and regions with its given diversity in socio economic status, health status, population dynamics, health system and infrastructure, health behavioral pattern, nutrition and food habit and also access to information and knowledge and other factors. Of which if we critically examine two of the very important factors like the i) population dynamics and CFR and ii) the health system ability and infrastructure with CFR to compare among countries which has been shown in the following figures (Fig: 1, 2, 3). Here in the different country age pyramids with an age segregated -cluster clearly depict/illustrate the fact that population constitutes and distribution by age and gender play a significant role in this disease risk, spread and, especially the difference in its pattern of age specific morbidity and mortality. ${ }^{11,14,15,16}$

Interestingly, there is a major difference in country wise spread, number case of Covid-19 and CFR. Perhaps one major difference is in distribution of world demography profile population age-clusters pyramids which explain the difference in impact of this pandemic. ${ }^{14,15}$

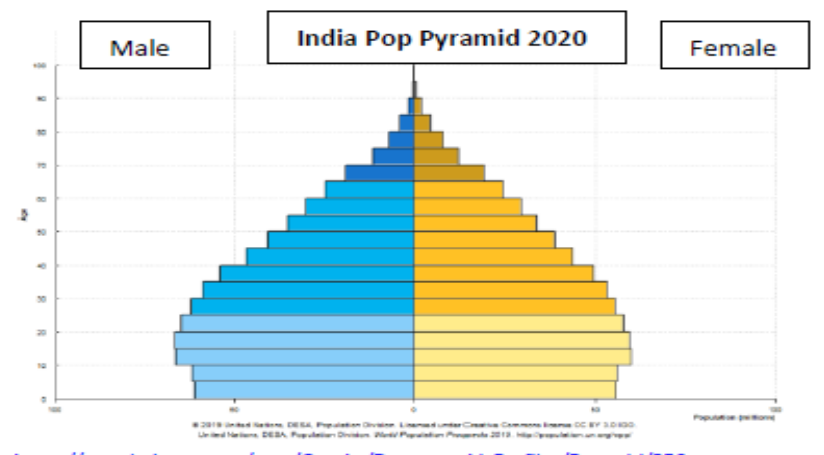

https://population.un.org/wpp/Graphs/DemographicProfiles/Pyramid/356

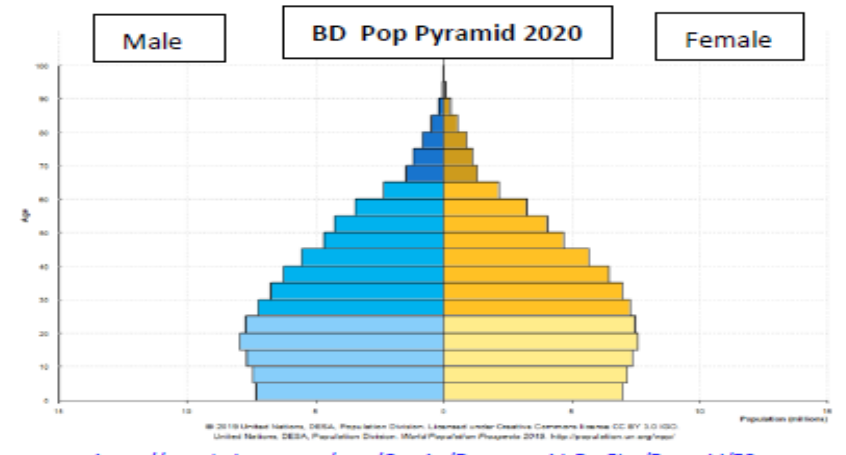

https://population.un.org/wpp/Graphs/DemographicProfiles/Pyramid/50

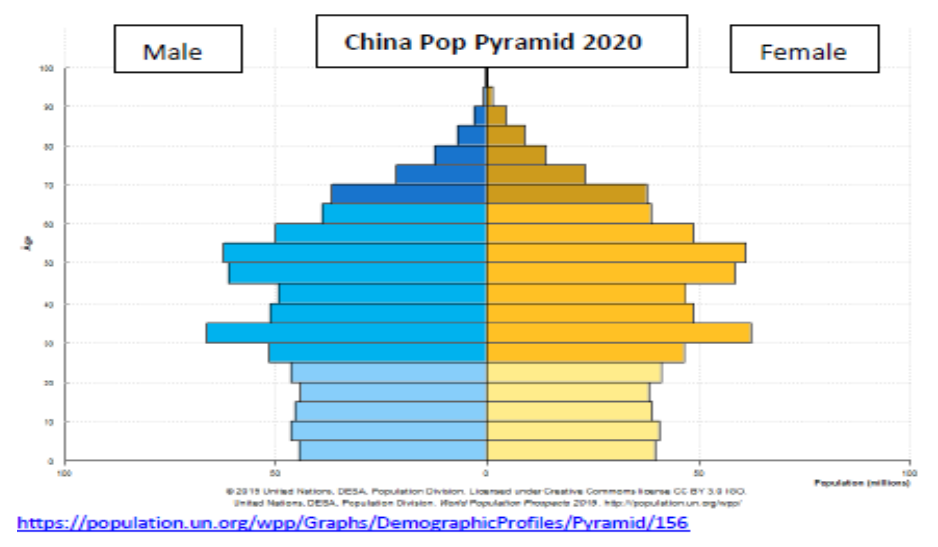

Figure : 1 
There is large variance across regions and countries in the capacity to cope with a sudden increase of serious cases that require intensive medical care and support, logistics, and sophisticated and scarce equipment. Differential access to the best health care facilities can also account for withincountry and within-region depending on socioeconomic condition, the other explanation points to the co-morbid health-condition and age composition of the population that contracted COVID-19 in each country. ${ }^{11,14,15,16}$ We can find elderly populations differ substantially between countries in terms of numbers of pre-existing conditions and/or severity of co-morbid conditions. ${ }^{11}$

In Bangladesh, distribution of demography profile population pyramids with age- clusters shows that in Bangladesh age above of 65 years is $5.1 \%$ (WB2018) (median age is 27.6 years, worldometer 2020 ), in case of India age above of 65years is $6.1 \%$ (WB2018) (The median age is 28.4 years, worldometer 2020) and in China age above of 65years is $10.9 \%$ (WB2018) (median age is 38.4 years (worldometer. 2020). So, we see less Covid-19 reported CFR in this part of the world among people above $65 .^{14,15,16}$

Whereas, in Italy, distribution of demography profile population pyramids with age- clusters shows that in Italy age above of 65years is $22.75 \%$ (WB2018) (median age is 47.3 years, worldometer 2020), in case of Spain age above of 65years is $19.37 \%$ (WB2018) (The median age is 44.9 years, worldometer 2020). It has been scientifically documented and observed that the people of age 65 and above usually suffer from one or more comorbid illness and are also at high risk and vulnerability.

The distribution of UK, demography profile population pyramids with age- clusters shows that in UK age above of 65years is $18.3 \%$ (WB2018) (median age is 40.5 years, worldometer 2020), in case of USA age above of 65years is 15.8\% (WB2018) (The median age is 38.3 years, worldometer 2020) ${ }^{14,15,16}$. Thereby, we can see daily huge increase of Covid-19 reported cases and jump in CFR which may not only be reasoned to public health inability rather a population dynamics associated to this. But here strongly to say, the Public health policies for non-pharmacological interventions are very important till treatment and vaccines are available.
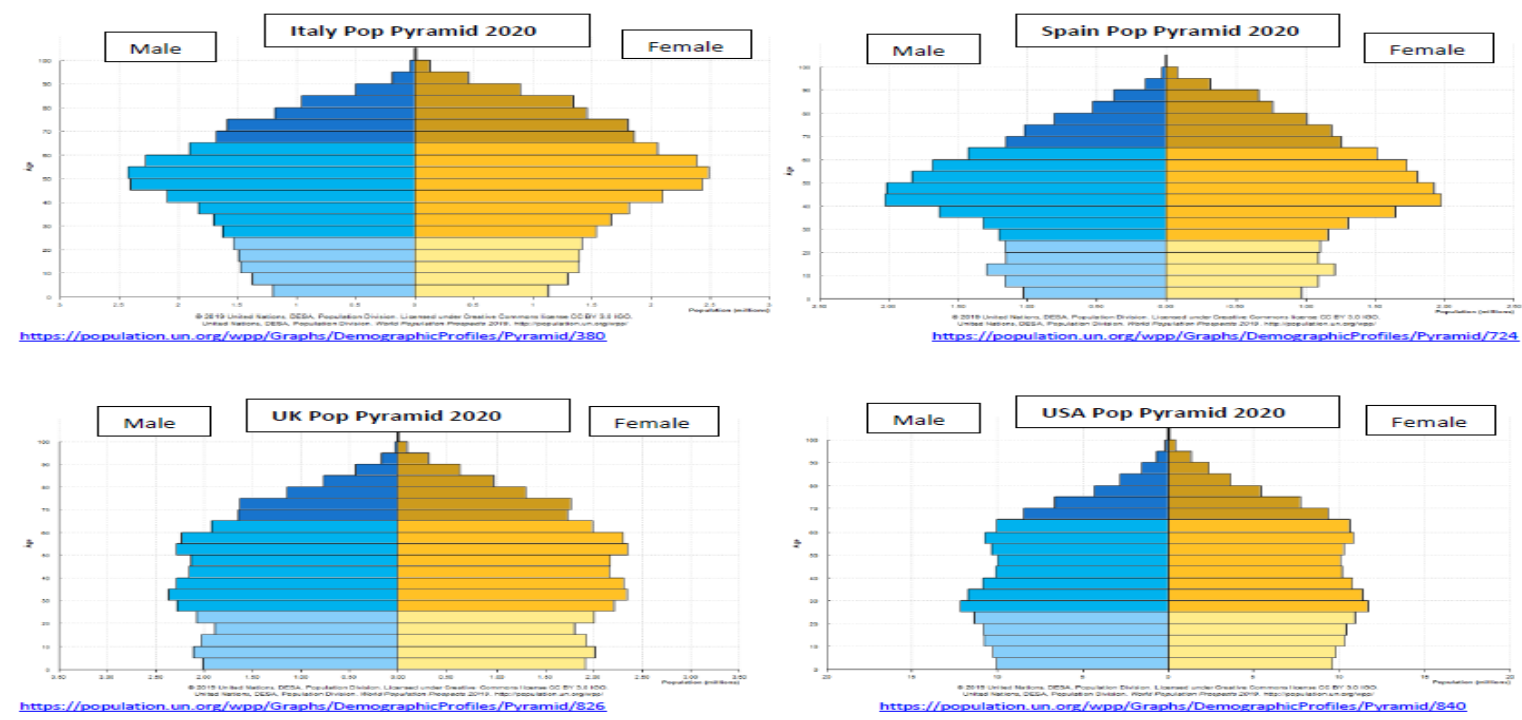

Figure : 2 
To look into the CFR and disease spread in relevance to country health system ability, capacity and infrastructure point of view, here if we see the global data, China and South Asian countries have low number of cases and CFR but on the other hand it's very high in Europe an USA. Health system ability of any country to provide health management support also influences mortality rates of a county as well. ${ }^{16,17}$

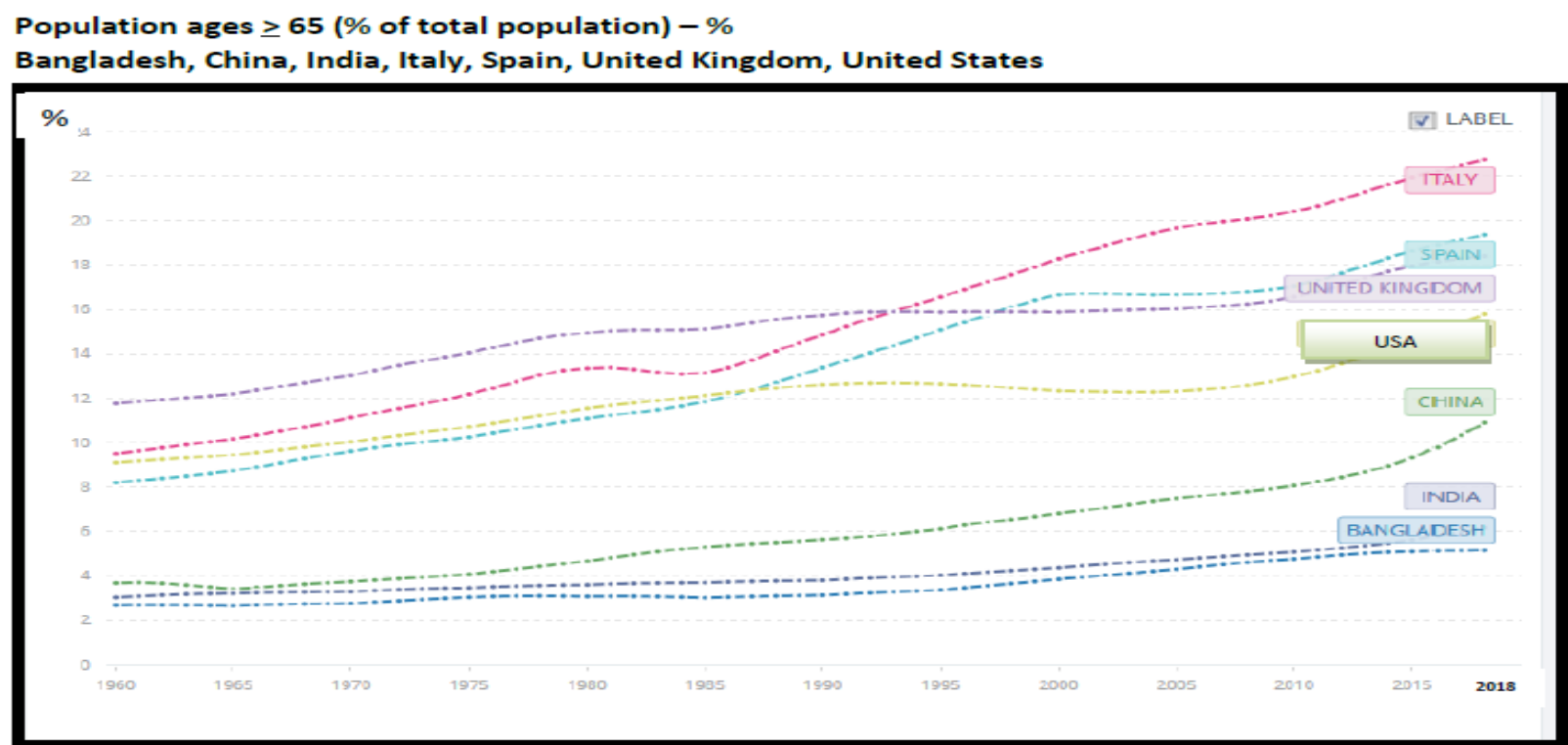

The World Bank, Data, https://data.worldbank.org/indicator/SP.POP.TOTL

Figure: 3

At the moment with the growing number of test and diagnosis, the symptomatic cases are surfacing as the tip of the iceberg, however there are huge number of both symptomatic and asymptomatic cases are yet to be identified with more expanded test facilities availability with time ahead. Doing more and more test will tell us about the county's total case numbers but in the developing countries most are 'mild cases' as described before. RT-PCR test is the most commonly used and reliable test for diagnosis of COVID-19. Serological diagnosis of antibodies of COVID-19 infection can be detected indirectly by host immune response to SARS CoV-2 infection, beyond the illness onset of first 2 weeks. It's an important tool to understand the extent of COVID-19 infection at the community level and to identify individuals who are already infected maybe immune and potentially "protected" and is especially important for peoples who were with mild to moderate illness. For serological diagnosis of COVID-19, IgM and IgG antibody ELISA-based tests are done. IgM and IgG seroconversion occurred in patients between the third and fourth week ${ }^{18,19,20 .}$

Finding epidemiological and public health solution to address this pandemic till today, experiences are widely varied and mixed in nature and the success story to slow down and containing the epidemic also varying across the globe. If we look into the public health remedy and solution as of now to deal with this COVID-19 disease, the Public health policy for non-pharmacological interventions includes proper diagnosis, contact tracing and isolation, hospital \& health services 
readiness and personal protections (PPEs). At individual level awareness and motivation, Community quarantine (Lockdowns), social/personal distancing, hand hygiene and personal hygiene and use of face mask, are important effective preventive and control measures. ${ }^{21,22}$. These measures may differ from country to country as per public health policy, population density and country economic status. But in case of Covid-19 the 'Group Risk' factors and age more than >65 years' agecluster is perhaps the most important influencing factor for case numbers and CFR in different countries around the globe.

There are more than dozens of anti-viral agents under trial for developing treatment of Covid-19 but only Remdesivir is showing some hope, but then we still have to wait for the result of the WHO 'Solidarity multi-country multi-centre clinical trial' to have an effective anti-viral(s). ${ }^{23,24}$ Vaccine is also 12-18 months away, about 100-vaccine trials are underway with hope for further prevention \& control of not only health burden but also recover from economic crisis. ${ }^{25,26}$

\section{Conclusion:}

In this $21^{\text {st }}$ century era COVID-19 is one of the fast spreading global pandemic of infectious disease origin which has also created high level concern among Public health and scientific community including business community, civil society and political leaders of the countries. The first index case was diagnosed in Wuhan, China ( Dec 2019) and within few months the global upsurge of cases obligated WHO to declare this as a disease of global Public health emergency .The disease varies significantly with its genomic sequence of virus pattern, sign, symptoms, characteristics including its epidemiological and public health responses across the countries. Also with the progression of the disease with its everyday new appearance of sign symptoms which are uniquely portraying this disease more of a kind of "COVID-19 syndrome" rather than as COVID-19. Along with many other factors associated to case fatality rate (CFR) as evidenced so far, the population dynamics and the health system infrastructure and ability factors are also some way influencing cases which are portrayed in this paper. The public health prevention practice and interventions demonstrate the total world is quite connected and also learning from each other's public health experiences to fight for the virus. However, the world community is eagerly waiting to see the result and outcome of the ongoing therapeutics and vaccine trial in several countries, the people of the world are quite optimistic and hopeful that, global scientific community will be able to invent some miraculous and magic solution in upcoming months which ultimately will free the world from this most terrifying COVID-19 pandemic of $21^{\text {st }}$ century.

\section{Reference:}

1. John Hopkins University. 2020. The Spread of the Coronavirus Outside China. Available at https://www.statista.com/chart/20935/covid-19-coronavirus-casesoutside-china/. Last accessed on 18th February 2020. 
2. DW Akademie 2020. Why a coronavirus upsurge could be devastating for South Asia. Available at https://www.dw.com/en/why-a-coronavirus-upsurge-could-bedevastating-for-south-asia/a-52233458. Last accessed on February 27, 2020.

3. Coronavirus disease (COVID-19) Situation Report, 2020 - 104 Data as received by WHO from national authorities by 10:00 CEST, 3 May 2020

4. World Health Organization (WHO). 2020c. Emergency Health Cluster Meeting on Novel Corona Virus, COVID-19. Available at https://www.who.int/bangladesh/news/detail/13-02-2020-emergency-health-clustermeeting-on-novel-corona-virus-covid-19. Last accessed on 13th February 2020.

5. David Mikkelson, Have Plagues Repeated Exactly Every 100 Years? Published 7 April 202 https://www.snopes.com/fact-check/plague-repeat-century/

6. David M. Morens, Jeffery K. Taubenberger, 2018, The Mother of All Pandemics Is 100 Years Old (and Going Strong)! Am J Public Health. 2018 November; 108(11): 1449-1454

7. https://www.theguardian.com/world/2020/apr/21/coronavirus-five-months-on-whatscientists-know-about-covid-19

8. https://www.cam.ac.uk/research/news/covid-19-genetic-network-analysis-providessnapshot-of-pandemic-origins

9. Graeme M. Lipper, MD , 2020, COVID Toes' and 'Kawasaki' Rash: 5 Cutaneous Signs in COVID-19, May 08, 2020

10. Diangeng Li, Meiling Jin, Pengtao Bao, Weiguo Zhao, Shixi Zhang, 2020, Clinical Characteristics and Results of Semen Tests Among Men With Coronavirus Disease 2019. Research Letter, Infectious Diseases. JAMA Network Open. 2020;3(5):e208292.

11. Alberto Palloni (CSIC) \& Stephan Walter (Rey Juan Carlos University), Policy Insights, COVID-19: How can we explain differences in mortality? by (https://population-europe.eu/policy-insights/covid-19-how-can-we-explaindifferences-mortality)

12. Monica Gandhi, M.D., M.P.H., Deborah S. Yokoe, M.D., M.P.H., and Diane V. Havlir, M.D. 2020 , Asymptomatic Transmission, the Achilles' Heel of Current Strategies to Control Covid-19, nejm.org on May 29, 2020

13. Neha Pathak, 2020 ,The Great Invader: How COVID-19 Attacks Every Organ.. Medscape infectious disease. Apr 23, 2020.

14. UN World Population prospects 2019 (https://population.un.org/wpp/Graphs/DemographicProfiles/Pyramid)

15. Tomáš Sobotka, Zuzanna Brzozowska, Raya Muttarak, Kryštof Zeman, Vanessa di Lego, 2020, Age, gender and COVID-19 infections: doi: https://doi.org/10.1101/2020.05.24.20111765

16. The World Bank, Data, https://data.worldbank.org/indicator/SP.POP.TOTL 
17. Margaret E Kruk, Michael Myers, S Tornorlah Varpilah, Bernice T Dahn . 2015, What is a resilient health system? Lessons from Ebola Lancet 2015; 385: 1910-12

18. https://www.medscape.com/viewarticle/927952? nlid $=134824 \_3044 \& s r c=W N L \_m d p l$ snews_200403_mscpedit_diab\&uac=97580EK\&spon=22\&impID=2334092\&faf $=1$ \# $v p \_2$

19. N Sethuraman, S S Jeremiah, ARyo, 2020, Interpreting Diagnostic Tests for SARSCoV-2.. JAMA Published online May 6,2020, Viewpoint, E1-E3.

20. Xiang $F$, Wang X, He X, et al. 2020, Antibody detection and dynamic characteristics in patients with COVID-19. Clin Infect Dis. 2020; ciaa461. Published online April 19, 2020. doi:10.1093/cid/ciaa461PubMedGoogle Scholar

21. Report 12 - The global impact of COVID-19 and strategies for mitigation and suppression | Faculty of Medicine | Imperial College London

22. WHO, 2020. https://www.who.int/emergencies/diseases/novel-coronavirus2019/question-and-answers-hub/q-a-detail/q-a-coronaviruses

23. Which Drugs Are Used Most for COVID-19 in Hospitals? - Medscape - Apr 30, 2020.

24. Nearly a Dozen Approved Drugs Could Be Effective Against COVID-19: Study Medscape - Apr 30, 2020.

25. Tung Thanh Le, Zacharias Andreadakis, Arun Kumar, Raúl Gómez Román, Stig Tollefsen, Melanie Saville and Stephen Mayhew, 2020 : The COVID-19 vaccine development landscape :Nature Reviews | Drug DISCOVERY, volume 19| May 2020 | 305

26. $N$. Zhang et al., 2020, Current development of COVID-19 diagnostics, vaccines and therapeutics, Microbes and Infection,https://doi.org/10.1016/j.micinf.2020.05.001 